\title{
Regression Optimal Functional Control for a Kind of Unsymmetrical System
}

\author{
Xixi Yue, Baili Su* \\ College of Engineering, Qufu Normal University, Rizhao, China \\ Email: *subaili111@126.com
}

How to cite this paper: Yue, X.X. and $\mathrm{Su}$ B.L. (2020) Regression Optimal Functional Control for a Kind of Unsymmetrical System. Applied Mathematics, 11, 363-376. https://doi.org/10.4236/am.2020.115026

Received: April 5, 2020

Accepted: May 10, 2020

Published: May 13, 2020

Copyright $\odot 2020$ by author(s) and Scientific Research Publishing Inc. This work is licensed under the Creative Commons Attribution International License (CC BY 4.0).

http://creativecommons.org/licenses/by/4.0/

\section{(c) (i) Open Acces}

\begin{abstract}
Because of the widespread existence of unsymmetrical system in the production process, its research is getting more and more attention. In this paper, a regression optimal functional control method is proposed for a class of unsymmetrical system. For the positive-negative model of the unsymmetrical system, a regression optimal functional controller is designed, which can make the system stable. The proposed algorithm has less computation and good control effect. Finally, three simulation examples are given to verify the effectiveness of the proposed algorithm.
\end{abstract}

\section{Keywords}

Unsymmetrical System, Positive-Negative Model, Basis Functions, Regression Optimal Functional Control

\section{Introduction}

As the most promising control theory, nonlinear system control theory has become the theme of modern control theory [1]. As a special kind of nonlinear system, the unsymmetrical system exists generally in modern industry, such as heating system, chemical system and mechanical system. The research on unsymmetrical system has attracted the attention of scholars since it was first proposed by Godfrey through steam experiment [2]. The characteristic of the unsymmetrical system is: the dynamic response of the system is completely different when the control input increases and decreases [3] [4] [5]. For the unsymmetrical system, if the controller is designed for a single linearized model of the whole system, the control effect is difficult to guarantee. At present, the study of unsymmetrical system has made some results. The modeling problem of unsymmetrical system is considered through an electronic nose system in [6]. Mul- 
tiple identification methods and linearization methods have been proposed to approximate the unsymmetrical system in [7].

Most of the early research about unsymmetrical system focus on system linearization, but pay less attention to the design of control algorithms. With the advantages of multi-step prediction, rolling optimization and feedback correction, Regression Optimal Control (ROC) [8] [9] has made great contributions in dealing with problems of nonlinear constraints, system parameter uncertainty and actuator saturation [10] [11] [12] [13]. Based on the above studies, the regression optimal control algorithm is designed for the positive-negative model of the unsymmetrical system, and the control effect is obviously better than a single linearized model control of the whole system in [14] [15]. The regression optimal control remedies the error caused by the linearization process, but it is difficult to obtain the analytical solution of the controller.

As one of the classic ROC algorithms, Regression Optimal Functional Control (ROFC) has received wide attention. Different from other control algorithms, regression optimal functional control algorithm designs the controller as a linear combination of known basis functions, and the analytic solution of the controller can be obtained easily [16]. In [17], the regression optimal functional control based on multi-model switching is proposed, which greatly reduces the calculation and achieves good control effects. In [18], a Proportional Integral Derivative (PID) control algorithm based on regression optimal functional control idea is proposed, which not only contains the advantages of the regression optimal functional control algorithm, but also has the simple structure of the traditional PID controller. Considering that the calculation of ROFC is small and the analytical solution is easy to obtain, a regression optimal functional control algorithm for unsymmetrical system is proposed in this paper.

At the end of this section, the main contributions of this paper are summarized: (a) The regression optimal functional control algorithm is applied to unsymmetrical system. (b) The analytical solution of the controller is obtained by using the Minimum Principle.

\section{Problem Statement and Preliminaries}

Consider a class of unsymmetrical system:

$$
\left\{\begin{array}{l}
x(k+1)=f^{\sigma}(x(k), u(k)) \\
y(k)=h^{\sigma}(x(k))
\end{array},\right.
$$

where $x(k) \in R^{n}$ is the system state, $u(k) \in R^{m}$ is the control input, $y(k) \in R^{q}$ is the system output. The control input $u(k)$ satisfies the following condition: $\|u(k)\|_{2} \leq u_{\max },\|\cdot\|$ denotes the Euclidean norm. $f$ and $h$ are smooth functions with appropriate dimensions. And the determining function of the positive-negative model is given as follows:

$$
\sigma=\left\{\begin{array}{l}
+, \text { if } u(k) \text { increases } \\
-, \text { if } u(k) \text { decreases }
\end{array},\right.
$$


This paper focuses on the control algorithm design for unsymmetrical system, so one of the linearization method in [15] is considered to obtain a linear representation of the unsymmetrical system. It is assumed that the origin is the equilibrium point of the system and that $f$ and $h$ are continuously differentiable at the origin. Expand the unsymmetrical system (1) at equilibrium point:

$$
\left\{\begin{array}{l}
x(k+1)=\frac{\partial f^{\sigma}}{\partial x^{\mathrm{T}}} x(k)+\frac{\partial f^{\sigma}}{\partial u^{\mathrm{T}}} u(k) \\
y(k)=\frac{\partial h^{\sigma}}{\partial x^{\mathrm{T}}} x(k)
\end{array},\right.
$$

The above linearization method is considered to obtain a linear representation of the unsymmetrical system (1) in the positive and negative directions:

$$
\left\{\begin{array}{l}
x(k+1)=A^{\sigma} x(k)+B^{\sigma} u(k) \\
y(k)=C^{\sigma} x(k)
\end{array},\right.
$$

where $A^{\sigma}, B^{\sigma}$ and $C^{\sigma}$ are constant parameter matrices with appropriate dimensions.

According to the recursive relationship, regression optimal model (4) can be written as:

$$
\begin{aligned}
& {\left[\begin{array}{c}
x(k+1) \\
x(k+2) \\
\vdots \\
x(k+P)
\end{array}\right]=\left[\begin{array}{c}
A^{\sigma} \\
\left(A^{\sigma}\right)^{2} \\
\vdots \\
\left(A^{\sigma}\right)^{P}
\end{array}\right] x(k)}
\end{aligned}
$$

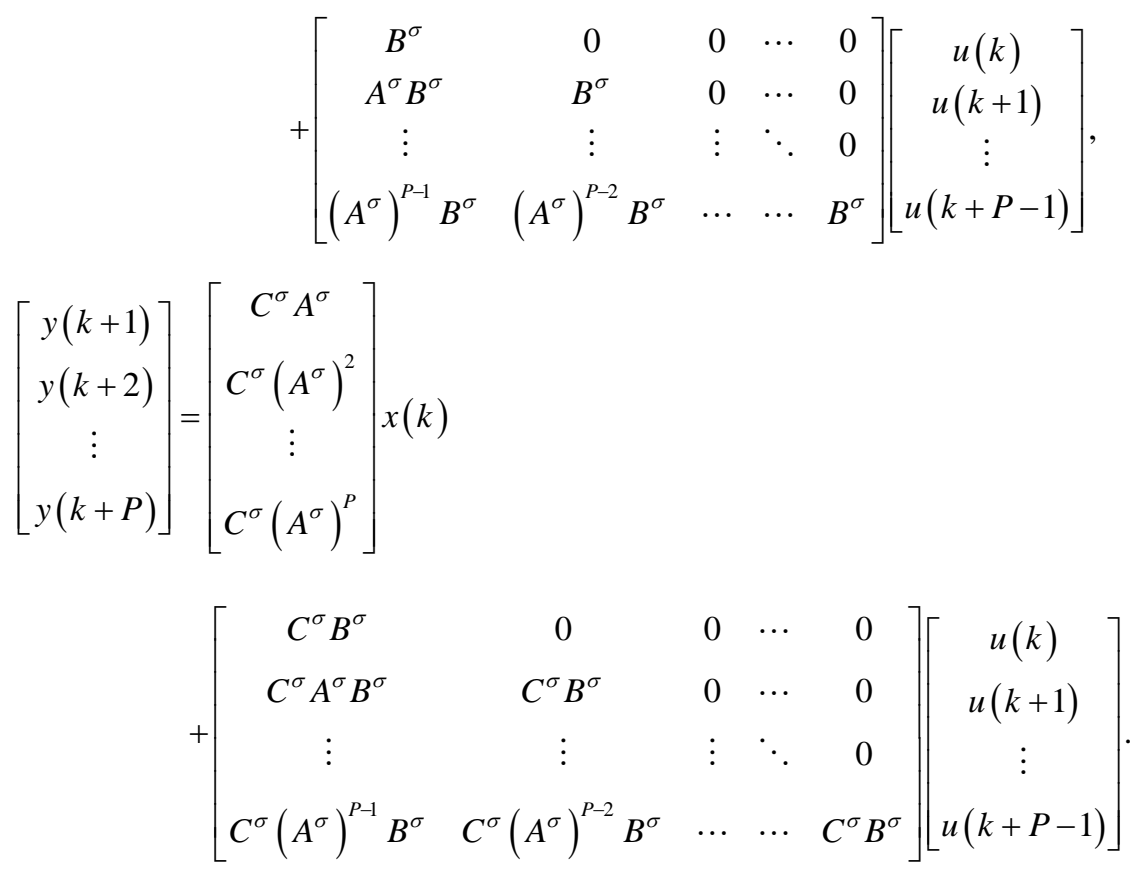

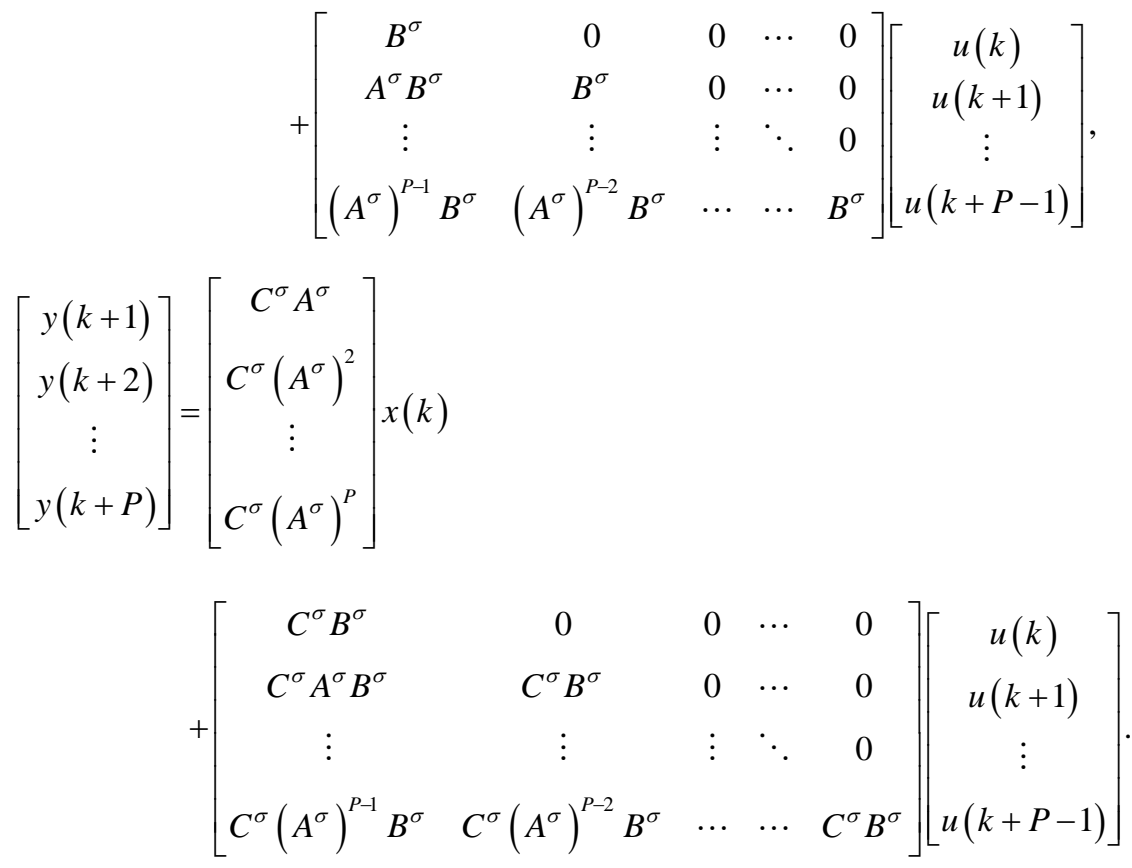


where $P$ is regression optimal step.

\section{Main Results}

\subsection{Theory of Regression Optimal Functional Control}

The design of the regression optimal functional control algorithm consists three steps as follows:

1) Multi-step prediction

Select appropriate regression optimal model for multi-step prediction:

$$
\left\{\begin{array}{l}
\hat{x}(k+1)=\hat{f}^{\sigma}(x(k), u(k)) \\
\hat{y}(k)=\hat{h}^{\sigma}(x(k))
\end{array},\right.
$$

2) Rolling optimization

Minimizing suitable performance indicator to calculate the control law:

$$
\min J^{\sigma}(k+j) \text { where } j=0,1, \cdots, P \text {. }
$$

By solving equation (8), we can get $u(k+j)$. Only $u(k)$ is applied to the system.

3) Feedback correction

At the next time $k+1$, according the error of the expected output and the systems output, the performance indicator $J$ is reoptimized to calculate $u(k+1)$.

\subsection{Design of Regression Optimal Functional Controller}

According to the idea of regression optimal functional control, we choose controller as:

$$
u^{\sigma}(k)=\sum_{l=1}^{L} \mu_{l}^{\sigma} g_{l}(k)
$$

where $\mu_{1}^{\sigma}$ is the weight coefficient of the linear combination of basis functions, $g_{l}$ is a set of known basis functions, $L$ is number of basis functions, $l=1,2, \cdots, L$.

Let $\Gamma^{\sigma}(k+i)=\left[\begin{array}{llll}\mu_{1}^{\sigma}(k+i) & \mu_{2}^{\sigma}(k+i) & \cdots & \mu_{L}^{\sigma}(k+i)\end{array}\right]$, $T(k+i)=\left[\begin{array}{llll}g_{1}(k+i) & g_{2}(k+i) & \cdots & g_{L}(k+i)\end{array}\right]^{\mathrm{T}}, \quad i=0,1, \cdots, P-1$.

Then, $u^{\sigma}(k+i)$ can be expressed as:

$$
\begin{aligned}
u^{\sigma}(k+i) & =\sum_{l=1}^{L} \mu_{l}^{\sigma}(k+i) g_{l}(k+i) \\
& =\Gamma^{\sigma}(k+i) T(k+i),
\end{aligned}
$$

Substituting Equation (10) into the regression optimal model (5) (6), and the regression optimal model can be written as:

$$
\left\{\begin{array}{l}
x_{m}=H^{\sigma} x(k)+\Gamma^{\sigma} \Psi^{\sigma} \\
y_{m}=F^{\sigma} x(k)+\Gamma^{\sigma} \Phi^{\sigma}
\end{array},\right.
$$

with 


$$
\begin{aligned}
& x_{m}=\left[\begin{array}{c}
x(k+1) \\
x(k+2) \\
\vdots \\
x(k+P)
\end{array}\right], y_{m}=\left[\begin{array}{c}
y(k+1) \\
y(k+2) \\
\vdots \\
y(k+P)
\end{array}\right], H^{\sigma}=\left[\begin{array}{c}
A^{\sigma} \\
\left(A^{\sigma}\right)^{2} \\
\vdots \\
\left(A^{\sigma}\right)^{P}
\end{array}\right], \\
& \Psi^{\sigma}=\left[\begin{array}{cccc}
\varphi_{11} & \varphi_{12} & \cdots & \varphi_{1 L} \\
\varphi_{21} & \varphi_{22} & \cdots & \varphi_{2 L} \\
\vdots & \vdots & \ddots & \vdots \\
\varphi_{L 1} & \varphi_{L 1} & \cdots & \varphi_{L L}
\end{array}\right], F^{\sigma}=C^{\sigma} H^{\sigma}, \Phi^{\sigma}=C^{\sigma} \Psi^{\sigma} .
\end{aligned}
$$

and

$$
\begin{gathered}
\varphi_{11}=B^{\sigma} g_{1}(k), \varphi_{12}=B^{\sigma} g_{2}(k), \varphi_{1 L}=B^{\sigma} g_{L}(k), \\
\varphi_{21}=A^{\sigma} B^{\sigma} g_{1}(k)+B^{\sigma} g_{1}(k+1), \\
\varphi_{22}=A^{\sigma} B^{\sigma} g_{2}(k)+B^{\sigma} g_{2}(k+1), \\
\varphi_{2 L}=A^{\sigma} B^{\sigma} g_{L}(k)+B^{\sigma} g_{L}(k+1), \\
\cdots \\
\varphi_{L 1}=\left(A^{\sigma}\right)^{P-1} B^{\sigma} g_{1}(k)+\left(A^{\sigma}\right)^{P-2} B^{\sigma} g_{1}(k+1)+\cdots+B^{\sigma} g_{1}(k+P-1), \\
\varphi_{L 2}=\left(A^{\sigma}\right)^{P-1} B^{\sigma} g_{2}(k)+\left(A^{\sigma}\right)^{P-2} B^{\sigma} g_{2}(k+1)+\cdots+B^{\sigma} g_{2}(k+P-1), \\
\varphi_{L L}=\left(A^{\sigma}\right)^{P-1} B^{\sigma} g_{L}(k)+\left(A^{\sigma}\right)^{P-2} B^{\sigma} g_{L}(k+1)+\cdots+B^{\sigma} g_{L}(k+P-1) .
\end{gathered}
$$

\subsection{Analysis of Performance Index}

According to regression optimal model (11), the expected output is defined as: $y_{r}=\left[\begin{array}{lllll}r^{\mathrm{T}}(k) & r^{\mathrm{T}}(k+1) & \ldots & r^{\mathrm{T}}(k+P)\end{array}\right]^{\mathrm{T}}$, the optimization problem of performance index is proposed:

$$
\begin{gathered}
\min J^{\sigma} \\
J^{\sigma}=\left(y_{r}-y_{m}\right)^{\mathrm{T}} Q\left(y_{r}-y_{m}\right)+\left(u^{\sigma}\right)^{\mathrm{T}} R u^{\sigma},
\end{gathered}
$$

where $Q=\operatorname{diag}\left[Q_{1}, Q_{2}, \cdots, Q_{P}\right]$ and $R=\operatorname{diag}\left[R_{1}, R_{2}, \cdots, R_{P}\right]$ are the weight matrices of appropriate dimensions, and $Q=Q^{\mathrm{T}}>0, R=R^{\mathrm{T}}>0$.

Substituting Equation (10), (11) into Equation (12):

$$
\begin{aligned}
J^{\sigma}=\left(y_{r}-y_{m}\right)^{\mathrm{T}} Q\left(y_{r}-y_{m}\right)+\left(u^{\sigma}\right)^{\mathrm{T}} R u^{\sigma} \\
=\left(y_{r}-\left(F^{\sigma} x(k)+\Gamma^{\sigma} \Phi^{\sigma}\right)\right)^{\mathrm{T}} Q\left(y_{r}-\left(F^{\sigma} x(k)+\Gamma^{\sigma} \Phi^{\sigma}\right)\right)+\left(\Gamma^{\sigma} T\right)^{\mathrm{T}} R\left(\Gamma^{\sigma} T\right) \\
=\left(y_{r}-F^{\sigma} x(k)\right)^{\mathrm{T}} Q\left(\left(y_{r}-F^{\sigma} x(k)\right)-2\left(\Gamma^{\sigma}\right)^{\mathrm{T}}\left(\Phi^{\sigma}\right)^{\mathrm{T}} Q\left(y_{r}-F^{\sigma} x(k)\right)\right) \\
+\left(\Gamma^{\sigma}\right)^{\mathrm{T}}\left(\left(\Phi^{\sigma}\right)^{\mathrm{T}} Q \Phi^{\sigma}+T^{\mathrm{T}} R T\right) \Gamma^{\sigma} .
\end{aligned}
$$

The necessary condition of minimum performance index is $\frac{\partial J^{\sigma}}{\partial u^{\sigma}}=0$, then: 


$$
\begin{gathered}
-2\left(\Phi^{\sigma}\right)^{\mathrm{T}} Q\left(y_{r}-F^{\sigma} x(k)\right)+2\left(\left(\Phi^{\sigma}\right)^{\mathrm{T}} Q \Phi^{\sigma}+T^{\mathrm{T}} R T\right) \Gamma^{\sigma}=0, \\
\Gamma^{\sigma}=\left(\left(\Phi^{\sigma}\right)^{\mathrm{T}} Q \Phi^{\sigma}+T^{\mathrm{T}} R T\right)^{-1}\left(\Phi^{\sigma}\right)^{\mathrm{T}} Q\left(y_{r}-F^{\sigma} x(k)\right),
\end{gathered}
$$

Then, we select that

$$
\begin{aligned}
& \mu_{1}^{\sigma}(k) \\
& =(1,0, \cdots, 0)\left(\left(\varphi_{11}\right)^{\mathrm{T}} Q_{1} \varphi_{11}+T^{\mathrm{T}}(k) R_{1} T(k)\right)^{-1}\left(\varphi_{11}\right)^{\mathrm{T}} Q_{1}\left(r(k)-C^{\sigma} A^{\sigma} x(k)\right) \\
& =\Theta_{r 1}^{\sigma} r(k)-\Theta_{x 1}^{\sigma} x(k), \\
& \mu_{2}^{\sigma}(k) \\
& =(0,1, \cdots, 0)\left(\left(\varphi_{12}\right)^{\mathrm{T}} Q_{1} \varphi_{12}+T^{\mathrm{T}}(k) R_{1} T(k)\right)^{-1}\left(\varphi_{12}\right)^{\mathrm{T}} Q_{1}\left(r(k)-C^{\sigma} A^{\sigma} x(k)\right) \\
& =\Theta_{r 2}^{\sigma} r(k)-\Theta_{x 2}^{\sigma} x(k), \\
& \mu_{L}^{\sigma}(k) \\
& =(0,0, \cdots, 1)\left(\left(\varphi_{1 L}\right)^{\mathrm{T}} Q_{1} \varphi_{1 L}+T^{\mathrm{T}}(k) R_{1} T(k)\right)^{-1}\left(\varphi_{1 L}\right)^{\mathrm{T}} Q_{1}\left(r(k)-C^{\sigma} A^{\sigma} x(k)\right) \\
& =\Theta_{r L}^{\sigma} r(k)-\Theta_{x L}^{\sigma} x(k) .
\end{aligned}
$$

And the current moments control input can be expressed as:

$$
\begin{aligned}
u^{\sigma}(k) & =\sum_{l=1}^{L} \mu_{l}^{\sigma}(k) g_{l}(k) \\
& =\sum_{l=1}^{L} \Theta_{r l}^{\sigma} r(k) g_{l}(k)-\sum_{l=1}^{L} \Theta_{x l}^{\sigma} x(k) g_{l}(k)
\end{aligned}
$$

\subsection{Design of Switching Law}

Define the increment of the control input as:

$$
\Delta u^{\sigma}(k)=u^{\sigma}(k)-u(k-1), \quad \sigma=+ \text { or }-
$$

The principle of the system selects controller is as follows

(1) If $\Delta u^{+}(k) \geq 0, \Delta u^{-}(k) \geq 0$, then $u(k)=u^{+}(k)$;

(2) If $\Delta u^{+}(k)<0, \Delta u^{-}(k)<0$, then $u(k)=u^{-}(k)$;

(3) If $\Delta u^{+}(k) \geq 0, \Delta u^{+}(k)<0$ or $\Delta u^{+}(k)<0, \Delta u^{+}(k) \geq 0$, then the system selects the controller that makes the performance index small: if $J^{+}<J^{-}$, then $u(k)=u^{+}(k)$; if $J^{+}>J^{-}$, then $u(k)=u^{-}(k)$.

Theorem 1. Consider unsymmetrical system (1), which can be approximated by positive-negative model (4), regression optimal functional controller (19) and switching law can ensure the closed loop system is stable.

Proof: According to the regression optimal functional control algorithm, the analytical solution of the controller $u^{\sigma}(k)$ is obtained by satisfying condition of $\frac{\partial J^{\sigma}}{\partial u^{\sigma}}=0$, which can ensure that the subsystems performance indicator $J^{\sigma}$ is minimized, respectively. 
If $u(k)=u^{+}(k) \quad\left(\right.$ or $u(k)=u^{-}(k)$ ), continue to calculate $u^{\sigma}(k+1)$ according to the above algorithm.

(1) When $\Delta u^{+}(k+1) \geq 0, \Delta u^{-}(k+1) \geq 0$, then $u(k+1)=u^{+}(k+1)$;

(2) When $\Delta u^{+}(k+1)<0, \Delta u^{-}(k+1)<0$, then $u(k+1)=u^{-}(k+1)$;

(3) When $\Delta u^{+}(k+1) \geq 0, \Delta u^{-}(k+1)<0$ or $\Delta u^{+}(k+1)<0$, $\Delta u^{-}(k+1) \geq 0$, the system selects the controller that makes the performance index smaller:

If

$$
\left(y_{r}-y_{m}\right)^{\mathrm{T}} Q\left(y_{r}-y_{m}\right)+\left(u^{+}\right)^{\mathrm{T}} R u^{+} \leq\left(y_{r}-y_{m}\right)^{\mathrm{T}} Q\left(y_{r}-y_{m}\right)+\left(u^{-}\right)^{\mathrm{T}} R u^{-},
$$

then $J^{+} \leq J^{-}, u(k)=u^{+}(k+1)$;

If

$$
\left(y_{r}-y_{m}\right)^{\mathrm{T}} Q\left(y_{r}-y_{m}\right)+\left(u^{+}\right)^{\mathrm{T}} R u^{+}>\left(y_{r}-y_{m}\right)^{\mathrm{T}} Q\left(y_{r}-y_{m}\right)+\left(u^{-}\right)^{\mathrm{T}} R u^{-},
$$

then $J^{+}>J^{-}, u(k)=u^{-}(k+1)$.

To sum up, when switching does not occur, the regression optimal controller can stabilize the system; when switching occurs, the switching law is designed according to the principle of positive and negative model matching. Under the constraint of switching law, the controller switches reasonably between positive controller and negative controller. Finally, the stability of the closed-loop system is guaranteed by feedback correction and rolling optimization.

The executive strategy of the regression optimal functional control algorithm in this paper is given as follows:

Step 1: When $k=1$, the analytical solution of $u^{\sigma}(k)$ is obtained by satisfying condition of $\frac{\partial J^{\sigma}}{\partial u^{\sigma}}=0$.

Step 2: $u(0)$ is given, define $\Delta u^{\sigma}(k)=u^{\sigma}(k)-u(k-1)$. According to the principle of selecting positive or negative controller, $u(k)=u^{+}(k)$ or $u(k)=u^{-}(k)$.

Step 3: If $u(k)=u^{+}(k)$ (or $\left.u(k)=u^{-}(k)\right)$, continue to calculate $u^{\sigma}(k+1)$ according to the above algorithm. (1) When $\Delta u^{+}(k+1) \geq 0, \Delta u^{-}(k+1) \geq 0$, then $u(k+1)=u^{+}(k+1)$; (2) When $\Delta u^{+}(k+1)<0, \Delta u^{-}(k+1)<0$, then $u(k+1)=u^{-}(k+1)$; (3) When $\Delta u^{+}(k+1) \geq 0, \Delta u^{-}(k+1)<0$ or $\Delta u^{+}(k+1)<0, \Delta u^{-}(k+1) \geq 0$, the system selects the controller that makes the performance index smaller: if $J^{+} \leq J^{-}$, then $u(k+1)=u^{+}(k+1)$; if $J^{+}>J^{-}$, then $u(k+1)=u^{-}(k+1)$.

Step 4: Let $k=k+2$, go to the Step 1 .

\section{Simulation Examples}

Consider three examples to verify the effectiveness of the algorithm in this paper:

Example 4.1 Consider the $p H$ control of acid-alkali neutralization with strong nonlinearity and strong unsymmetry. The neutral reaction of strong acid and 
alkali is conducted in the continuous stirring reactor. Assuming that the reaction level is stable, a mathematical model of the reaction process in CSTR is established:

$$
\left\{\begin{array}{l}
V \frac{\mathrm{d} x(t)}{\mathrm{d} t}=u(t)(b-x(t))-F(t)(a+x(t)) \\
p H(t)=\lg \frac{x(t)+\sqrt{x(t)^{2}+4 K_{w}}}{2 K_{w}}
\end{array},\right.
$$

where $u(t)$ is the flow rate of the alkaline solution, $F(t)$ is the flow rate of the acid solution, $V$ is the volume of the reactor, $x(t)$ is the difference between $\left[\mathrm{OH}^{-}\right]$and $\left[\mathrm{H}^{+}\right], a$ is the concentration of the acid solution and $b$ is the concentration of the alkaline solution, the water balance constant $K_{w}=10^{-14}$.

With the flow rate of the alkaline solution $u(t)$ as the control input and $p H$ value $p H(t)$ as the output variable, consider the system has only one equilibrium point, the sampling time $T_{s}=0.02 \mathrm{~s}$, establish the positive-negative model:

$$
\left\{\begin{array}{l}
x(k+1)=A^{\sigma} x(k)+B^{\sigma} u(k) \\
y(k)=C^{\sigma} x(k)
\end{array},\right.
$$

where $A^{\sigma}=1-\frac{T_{s}}{V} u_{0}-\frac{T_{s}}{V} F_{0}+k_{1}^{\sigma}, \quad B^{\sigma}=\frac{T_{s}}{V}\left(b-x_{0}\right)+k_{2}^{\sigma}, \quad C^{\sigma}=\frac{1}{\ln 10 \sqrt{x_{0}^{2}+4 K_{w}}}$, $u_{0}, x_{0}, F_{0}$ are the corresponding values of each variable in the equilibrium state. $\quad a=0.01 \mathrm{kmol} / \mathrm{L}, \quad b=0.01 \mathrm{kmol} / \mathrm{L}, \quad u_{0}=0.112 \mathrm{~L} / \mathrm{s}, \quad F_{0}=0.112 \mathrm{~L} / \mathrm{s}$, $x_{0}=0.009, V=0.02 \mathrm{~kL}, k_{1}^{\sigma}, k_{2}^{\sigma}$ are the correction factor of the positive-negative model, $k_{1}^{+}=1.84, k_{1}^{-}=-0.25, k_{2}^{+}=0.12, k_{2}^{-}=0.07$.

Select the wavelet functions as the basis functions of the regression optimization functional controller: $g(t)=\frac{2}{\sqrt{3 \sqrt{\pi}}}\left(1-t^{2}\right) \exp \left(-\frac{t^{2}}{2}\right)$. The output of the three basis functions is shown in Figure 1.

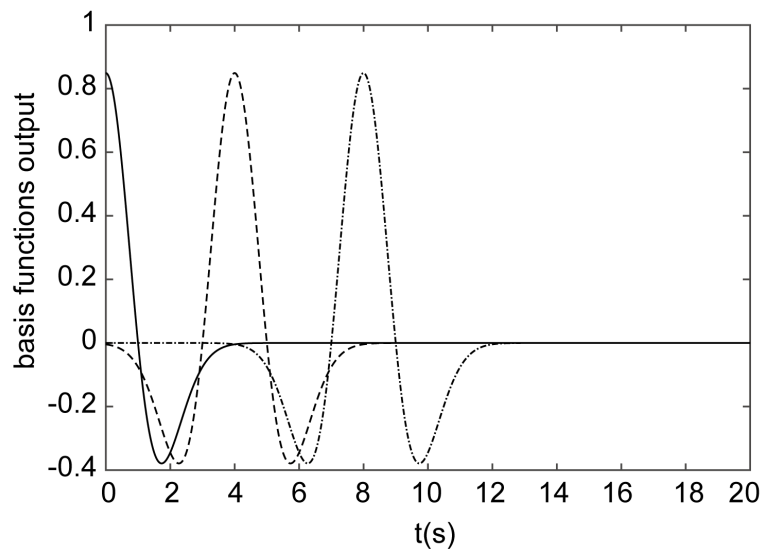

Figure 1. Basis functions output. 
The weight coefficients of the performance index $Q=I$ and $R=I$, regression optimal step $P=8$. The simulation results are shown in Figure 2, Figure 3.

The solid lines and the dotted lines represent the system response trajectories under regression optimal functional control and regression optimal control, respectively. When the system adopts the regression optimal functional control method, the system switches at $t=1.74 \mathrm{~s}$. When the system adopts the regression optimal control method, the system switches at $t=3.16 \mathrm{~s}$. Simulation results indicate that the regression optimal functional control algorithm can ensure the nonlinear unsymmetrical system state is ultimately stable. And compared with regression optimal controller, the regression optimal functional controller can bring the system state to steady faster.

Example 4.2 Consider a numerical example:

$$
\left\{\begin{array}{l}
\dot{x}=A^{\sigma} x+B^{\sigma} u \\
y=C^{\sigma} x
\end{array},\right.
$$

where

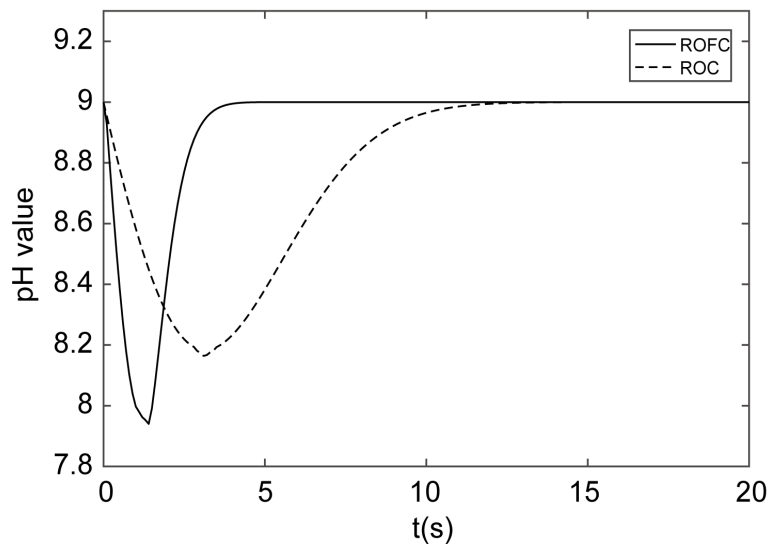

Figure 2. The trajectories of output.

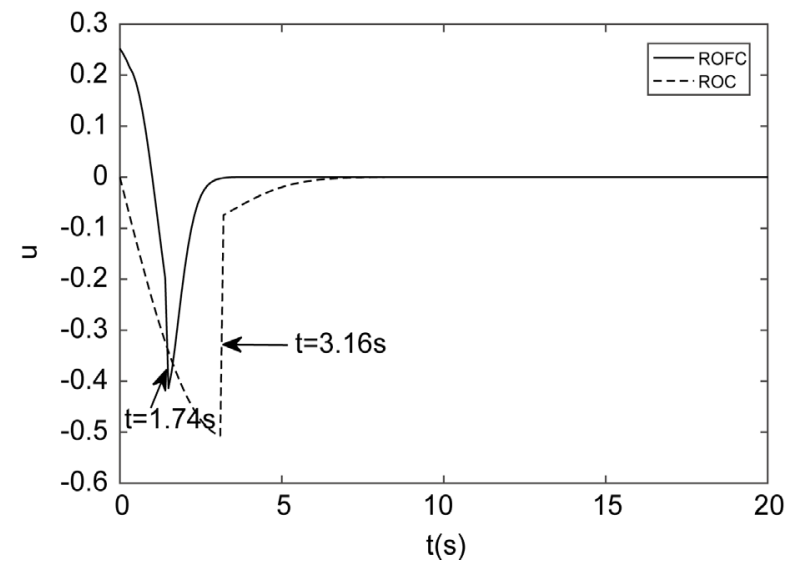

Figure 3. The trajectories of control input. 


$$
\begin{aligned}
& x=\left[\begin{array}{l}
x_{1} \\
x_{2}
\end{array}\right], A^{+}=\left[\begin{array}{ll}
4 & 0 \\
1 & 3
\end{array}\right], A^{-}=\left[\begin{array}{cc}
-4 & 0 \\
1 & -5
\end{array}\right], \\
& B^{+}=\left[\begin{array}{l}
1 \\
0
\end{array}\right], B^{-}=\left[\begin{array}{c}
-1 \\
0
\end{array}\right], C^{\sigma}=\left[\begin{array}{ll}
1 & 0
\end{array}\right] .
\end{aligned}
$$

Next, the positive-negative model is discretized, and the sampling time is $T_{s}=0.02 \mathrm{~s}$. And the weight coefficient matrices of the performance indexs $Q=I$ and $R=I$, regression optimal step $P=8$. Choose wavelet functions as basis functions like Example 4.1. The simulation results are shown in Figures 4-6:

The solid lines and the dotted lines represent the system response trajectories under regression optimal functional control and regression optimal control, respectively. Both two control methods make the system switches at $t=1.48 \mathrm{~s}$. The simulation results show that the regression optimal functional control algorithm can ensure the system state is ultimately stable. And compared with regression optimal controller, the regression optimal functional controller has better control effect.

Example 4.3 (Tracking Problem) The system in Example 4.2 is considered, and the sampling time is $T_{s}=0.02 \mathrm{~s}$, regression optimal step $P=8$, the weight

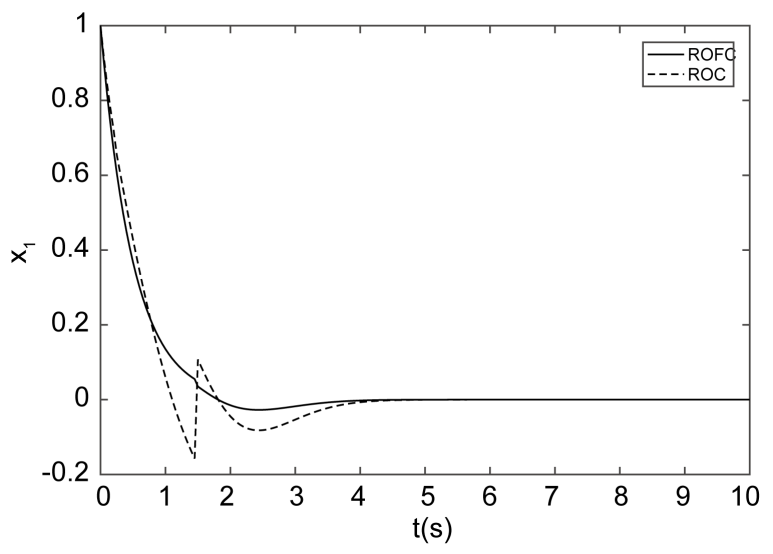

Figure 4. The trajectories of state $X_{1}$.

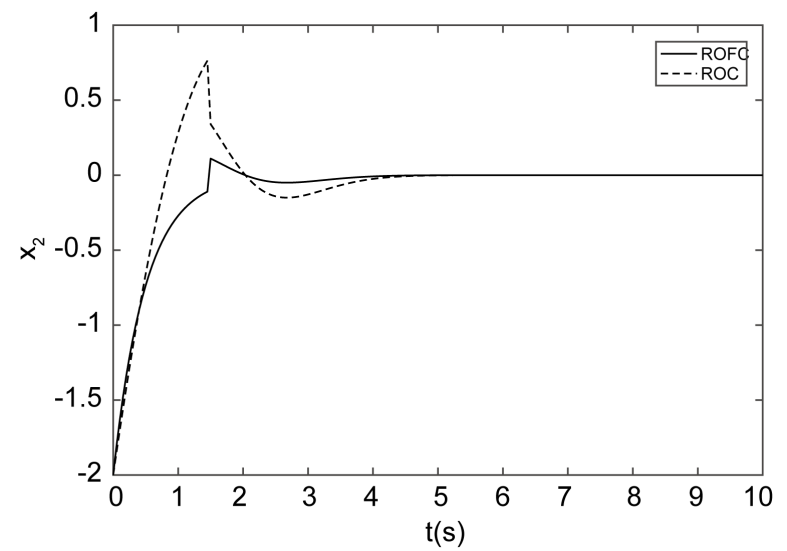

Figure 5. The trajectories of state $x_{2}$. 
coefficient matrices $Q=I$ and $R=I$. Choose wavelet functions as basis functions like Example 4.1. Let the expected output $y_{r}=\sin (t)$, and the simulation results are shown in Figures 7-10:

In Figure 7, the solid line and the dotted line represent the system output and the expected output, respectively. The system switches at $t=0.42 \mathrm{~s}, t=1.60 \mathrm{~s}$ and $t=3.02 \mathrm{~s}$. The simulation results show that the system under the regression

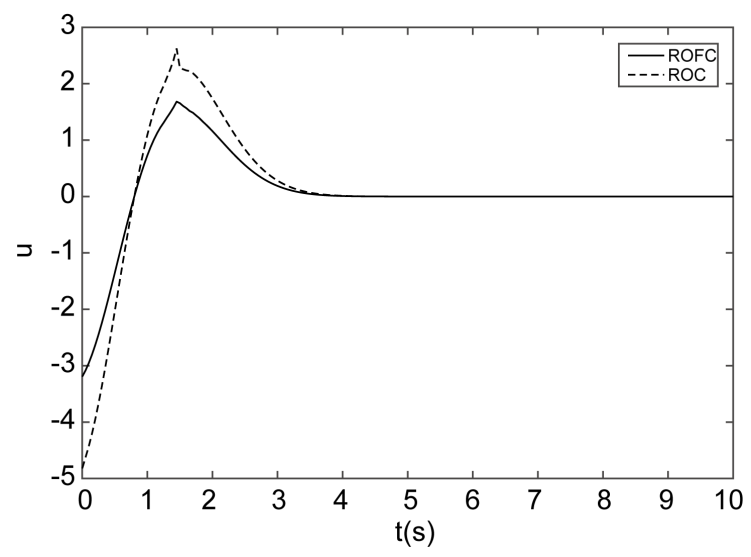

Figure 6. The trajectories of control input $u$.

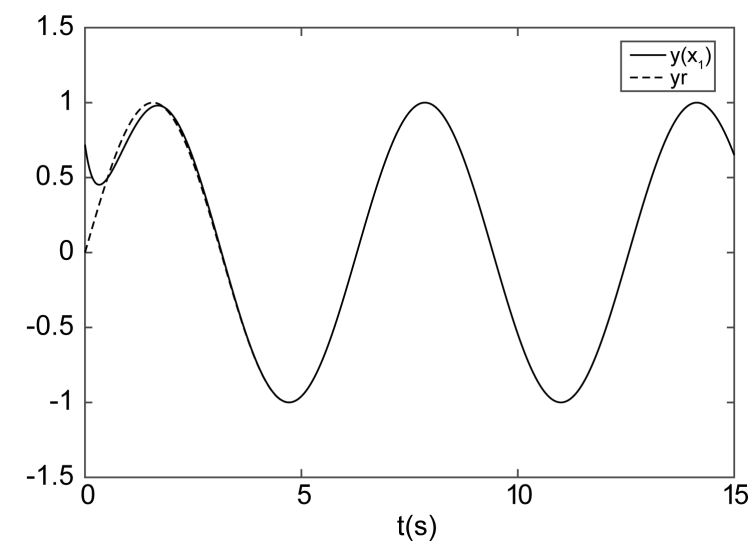

Figure 7. The trajectories of $y$ and $y_{r}$

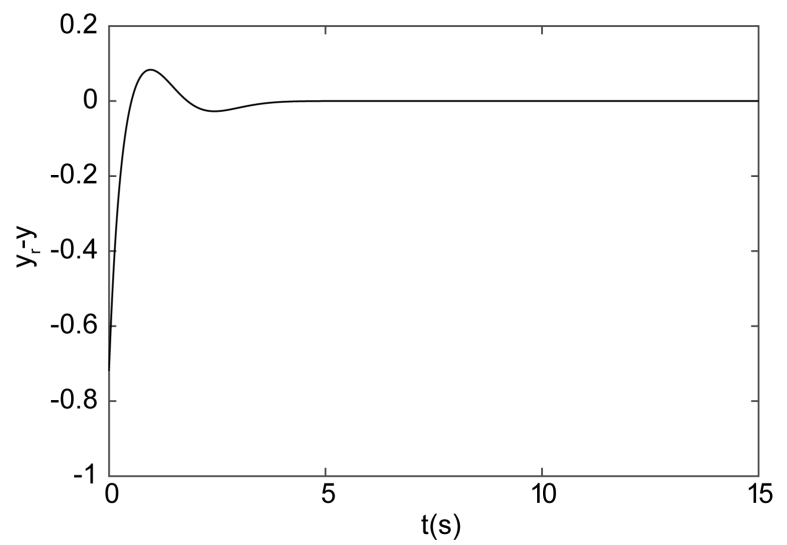

Figure 8. The trajectory of $y_{r}-y$. 


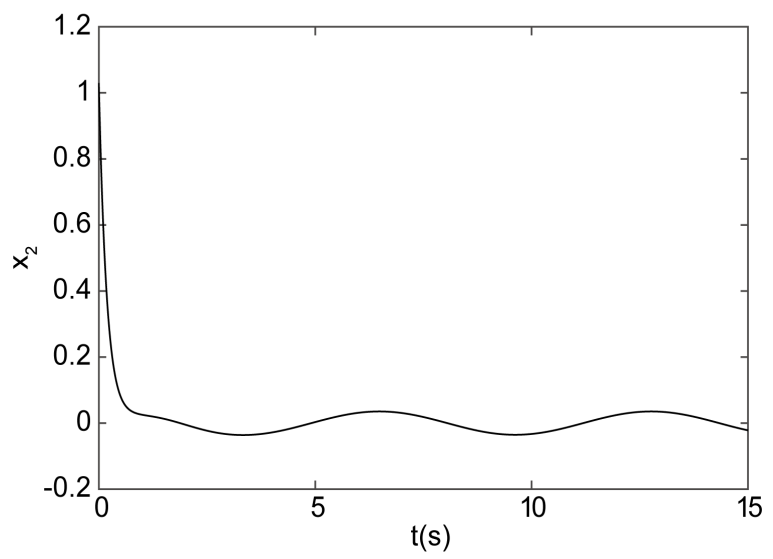

Figure 9. The trajectory of $x_{2}$.

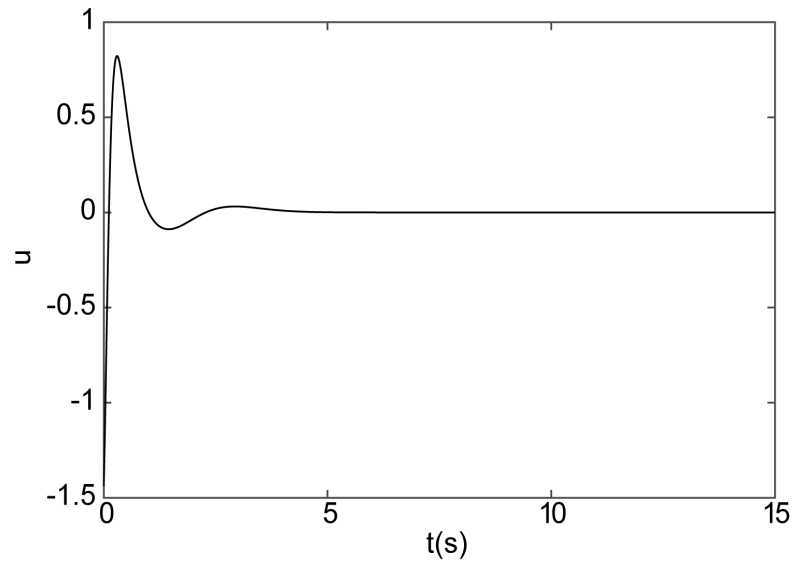

Figure 10. The trajectory of $u$.

optimal functional control can track the expected output in a short time.

\section{Conclusion}

In this paper, the regression optimal functional control algorithm for a class of unsymmetrical system is proposed. For the positive-negative model of unsymmetrical system, the regression optimal functional controller is designed. Based on the matching of the positive-negative model, the controller switches between positive controller and negative controller reasonably, which can make the system stable. The proposed algorithm has less computation and good control effect. Three simulation examples are used to verify the effectiveness of the proposed algorithm.

\section{Date Availability}

No data were used to support this study.

\section{Conflicts of Interest}

The authors declare that they have no conflicts of interest. 


\section{Acknowledgements}

This research was supported by the Natural Science Foundation of China (Grant Nos. 61374004, 61773237, 61473170).

\section{References}

[1] Jiang, C.S. and Wu, Q.X. (2012) Robust Control Basis of Modern Nonlinear Systems. Harbin Institute of Technology Press, Harbin.

[2] Godfrey, K.R. and Briggs, P.A.N. (1972) Identification of Processes with Direction-Dependent Dynamic Responses. IEE Proceedings of the Institution of Electrical Engineers, 119, 1733-1739. https://doi.org/10.1049/piee.1972.0350

[3] Wang, Q.G., Hang, C.C. and Zou, W. (1998) Automatic Tuning of Nonlinear PID Controllers for Unsymmetrical Processes. Computers and Chemical Engineering, 22, 687-694. https://doi.org/10.1016/S0098-1354(97)00220-2

[4] Turner, P., Montague, G. and Morris, J. (1996) Nonlinear and Direction-Dependent Dynamic Process Modelling Using Neural Networks. IEE Proceedings-Control Theory and Applications, 143, 44-48. https://doi.org/10.1049/ip-cta:19960061

[5] Tan, A.H. (2003) Identification of Direction-Dependent Processes Using Maximum Length Ternary Signals. IEE Proceedings-Control Theory and Applications, 152, 170-178. https://doi.org/10.1049/ip-cta:20030147

[6] Rosenqvist, F., Tan, A.H., Godfrey, K. and Karlstrom, A. (2006) Direction-Dependent System Modeling Approaches Exemplified through an Electronic Nose System. IEEE Transactions on Control Systems Technology, 14, 526-531.

https://doi.org/10.1109/TCST.2005.860520

[7] Tan, A.H. (2009) Direction-Dependent Systems: A Survey. Automatica, 45, 2729-2743. https://doi.org/10.1016/j.automatica.2009.09.024

[8] Xi, Y.G., Li, D.W. and Lin, S. (2013) Model Predictive Control: Status and Challenges. Journal of Automation, 39, 222-236. https://doi.org/10.1016/S1874-1029(13)60024-5

[9] Khajji, B., Labzai, A., Kouidere, A., Balatif, O. and Rachik, M. (2020) A Discrete Mathematical Modeling of the Influence of Alcohol Treatment Centers on the Drinking Dynamics Using Optimal Control. Journal of Applied Mathematics, 2020, Article ID: 9284698. https://doi.org/10.1155/2020/9284698

[10] Su, B.L., Li, S.Y. and Zhu, Q.M. (2009) The Design of Predictive Control with Characterized Set of Initial Condition for Constrained Switched Nonlinear System. Science in China Series E: Technological Sciences, 52, 456-466. https://doi.org/10.1007/s11431-008-0249-8

[11] Yan, Y. and Su, B.L. (2015) Design of Explicit Fuzzy Prediction Controller for Constrained Nonlinear Systems. Mathematical Problems in Engineering, 2015, Article ID: 857546. https://doi.org/10.1155/2015/857546

[12] Zhu, B. and Xia, X.H. (2016) Lyapunov-Based Adaptive Model Predictive Control for Unconstrained Non-Linear Systems with Parametric Uncertainties. IET Control Theory and Applications, 10, 1937-1943. https://doi.org/10.1049/iet-cta.2016.0203

[13] Su, B.L. and Chunyu, D.D. (2018) Finite-Time Optimization Stabilization for a Class of Constrained Switched Nonlinear Systems. Mathematical Problems in Engineering. https://doi.org/10.1155/2018/6824803

[14] Feng, A.X., Wei, M.H. and Luo, X.L. (2012) Model Predictive Control with Input Feedback and Its Controllability for Unsymmetrical Systems. CIESC Journal, 63, 
106-111.

[15] Luo, X.L., Wei, M.H., Xu, F. and Feng, A.X. (2013) Model Predictive Control for Nonlinear Unsymmetrical Systems. Control and Decision, 28, 763-768.

[16] Zhang, R.D. and Wang, S.Q. (2007) Adaptive Predictive Functional Control for a Class of Nonlinear Systems. Control and Decision, 6, 711-715.

[17] Xu, W., Zhang, J.F. and Zhang, R.D. (2017) Application of Multi-Model Switching Predictive Functional Control on the Temperature System of an Electric Heating Furnace. ISA Transactions, 68, 287-292. https://doi.org/10.1016/j.isatra.2017.02.001

[18] Zhang, J.M. (2017) Design of a New PID Controller Using Predictive Functional Control Optimization for Chamber Pressure in a Coke Furnace. ISA Transactions, 67, 208-214. https://doi.org/10.1016/j.isatra.2016.11.006 